\title{
Defect Formation and Kinetics of Atomic Terrace Merging
}

\author{
Ajay Gopinathan and T.A. Witten \\ Dept. of Physics and James Franck Institute, The University of Chicago, Chicago, IL 60637
}

(November 15, 2018)

\begin{abstract}
Pairs of atomic scale terraces on a single crystal metal surface can be made to merge controllably under suitable conditions to yield steps of double height and width. We study the effect of various physical parameters on the formation of defects in a kinetic model of step doubling. We treat this manifestly non- equilibrium problem by mapping the model onto a 1-D random sequential adsorption problem and solving this analytically. We also do simulations to check the validity of our treatment. We find that our treatment effectively captures the dynamic evolution and the final state of the surface morphology. We show that the number and nature of the defects formed is controlled by a single dimensionless parameter $q$. For $q$ close to one we show that the fraction of defects rises linearly with $\epsilon \equiv 1-q$ as $0.284 \times \epsilon$. We also show that one can arrive at the final state faster and with fewer defects by changing the parameter with time.
\end{abstract}

\section{INTRODUCTION}

Structural phase transitions on stepped metals have gained much attention in recent years. Starting from some of the first experiments by Lang et al [1] who noticed single height steps merging to form double height steps on Pt $\{544\}$ to time lapsed STM measurements of the dynamics of the step doubling process more recently $[2,3]$ the field has been attracting more attention. The study of surface structure and in particular the formation of defects during the step doubling process is important as these influence the desirable technological properties of the surfaces. Various experimental [2-5] and theoretical studies [6,7] have been performed to elucidate the dynamics of the evolution of these step doubling processes. However one major aspect that has been missing from the theoretical endeavours is to characterize the evolution of defects in these processes. One of our main motivations is to address this question theoretically.

An important characteristic of the step doubling process is that, under the optimal conditions, it is manifestly non-equilibrium. Steps seem to double irreversibly (unless conditions are changed) and hence equilibrium statistical mechanics treatments are suspect. The problem is thus a part of a whole set of interesting problems. These include non-equilibrium epitaxial growth models, many of which are described by the Kardar-Parisi-Zhang equation [8]. Irreversible depositon of macromolecules on surfaces and sociological epidemic models [9] also fall in this category. Our approach to treating the step doubling process is derived from treatments of random sequential adsorption (RSA) problems [10] which deal with irreversible adsorption of objects onto lattices.

In this paper we consider a simple model [11] for the step doubling process described by two parameters, a nucleation rate and a zippering rate. We first map this problem onto a one dimensional random sequential adsorption problem with two kinds of species adsorbing onto the lattice corresponding to perfectly doubled steps and defective structures. We then utilize the methods of RSA to solve the problem analytically. We are able to make predictions for the fraction of defects and perfectly doubled steps in the asymptotic stages of the process. We also predict the dynamical evolution of the degree to which the surface has undergone doubling. The theoretical predictions are then checked against extensive simulation results and found to be in good agreement. We finally make contact with experimental results and show how our analysis can be used to extract information about the dynamics of the process.

The paper is organized as follows. We first introduce our model for the step doubling process which utilizes a coarse grained picture of the surface. We then solve the problem in the special limit where the zippering rate is infinite by mapping it exactly to the problem of random sequential adsorption of dimers on a 1-D lattice. We then step back and set up the general mathematical framework for random sequential adsorption of more than one species. We then use this framework to tackle the problem of accounting for defects. We then generalize this to the case where our field of view does not encompass the whole sample but just a part of it, since this is closer to the experimental situation. We then discuss the time dependence of the surface morphology. Next we present our simulation methods and we finally conclude by discussing our results. 


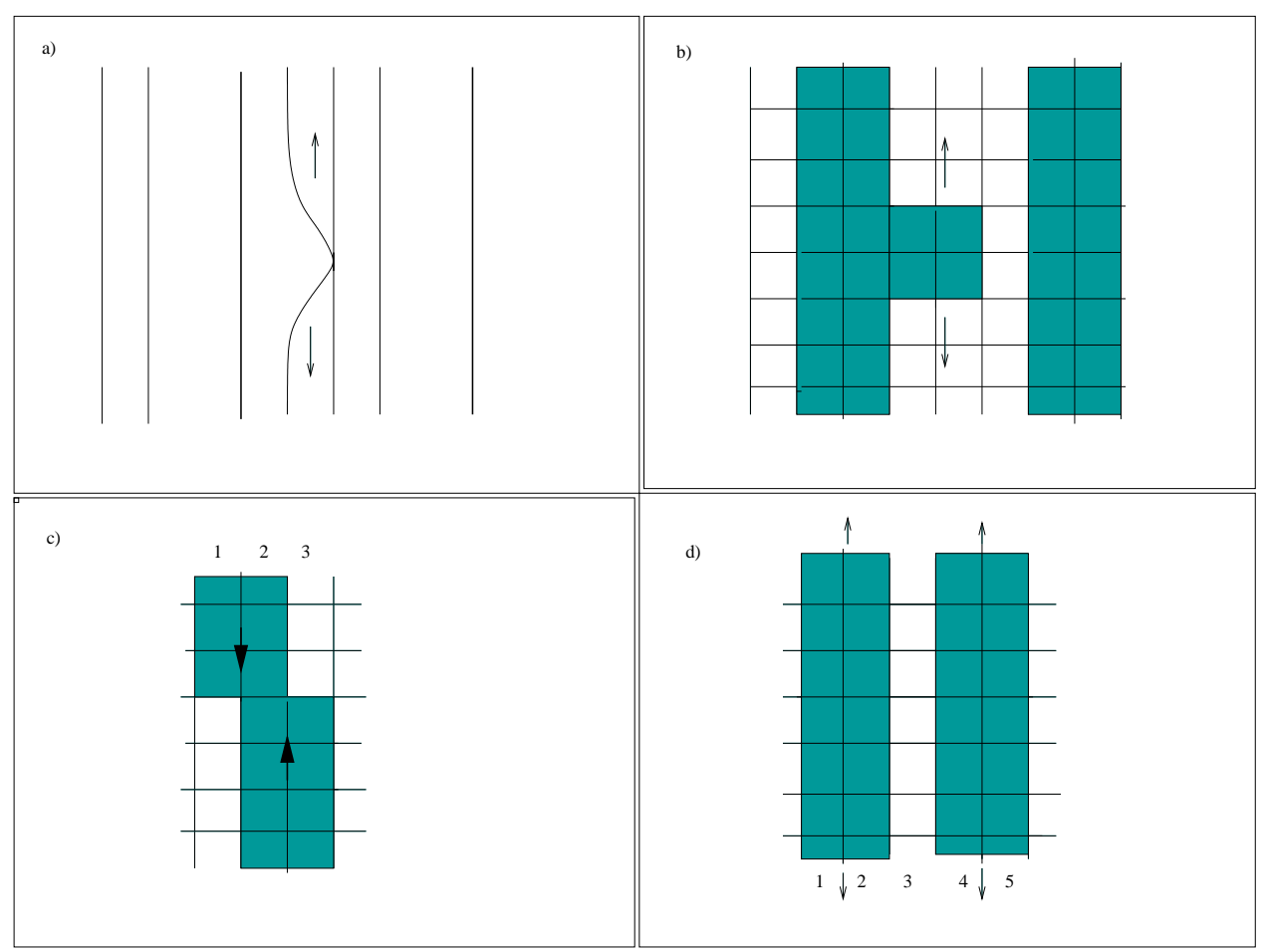

FIG. 1. (a) A schematic view of single and double steps. The lines represent step edges. The thick lines denote double step edges. The terrace on the left of an edge is higher than the one to the right. Also shown is a single step edge bulge touching its downstairs neighbor thus initiating a nucleation. The two edges will subsequently zip together, as indicated by the arrows, to form a double step. (b) A coarse grained lattice representation of the situation in (a). Shaded sites represent occupied sites i.e. sites belonging to a double step.(c) A frustrated dead end forms when steps 1 and 2 attempt to double and steps 2 and 3 also attempt to double starting at another nucleation point. (d) An isolated step is formed when steps 1 and 2 and steps 4 and 5 double leaving step 3 with no partner to double with.

\section{THEORETICAL ANALYSIS}

It is found that steps commence coalescing at a point contact where a step edge bulge touches the neighboring step edge below it $[2,3]$ (see fig. 1). This is called a nucleation event. Once a nucleus has been formed the two steps begin coalescing steadily in both directions from the nucleus. This process is referred to as zippering. Thus there are two distinct rates that govern the formation of the doubled structure, the nucleation rate $I$ (defined as the number of nucleations that occur per unit time on a sample that has no doubled steps) and the zippering rate $Z$ (defined as the rate of change of the length of a doubled step in units of the step width). This zippering rate is observed to be large enough that many steps undergo complete doubling. Our analysis will focus on this regime of small $I / Z$.

When the step doubling process has reached its final state two main kinds of defects are observed to remain. The first kind are isolated step edges whose neighbors to both the right and left have doubled with their other neighbors leaving the step "isolated" (see fig. 1). The second kind are frustrated dead ends which are formed when a step merges to its left at one point along the step and to its right at another point. We wish to understand how the nucleation and zippering rates affect the formation of these defects.

\section{A. Final State}

The simplest model assumes that nucleation points occur randomly over the entire sample with a probability per unit time determined by the nucleation rate $(I)$. The steps then coalesce at a steady rate $(Z)$. To attack this problem we first imagine mapping a square section of the sample to a coarse grained lattice (see fig. 1). Here each column represents a step. A lattice site can either be occupied or unoccupied. Occupied sites represent sections of a step that have doubled. A step is allowed to double only with its downstairs neighbor (to the right as in fig. 1). Thus occupied lattice sites occur in pairs, one each on adjacent steps. A nucleation event where a step edge bulge touches 


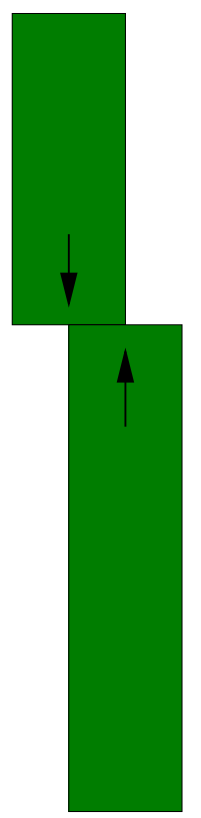

B

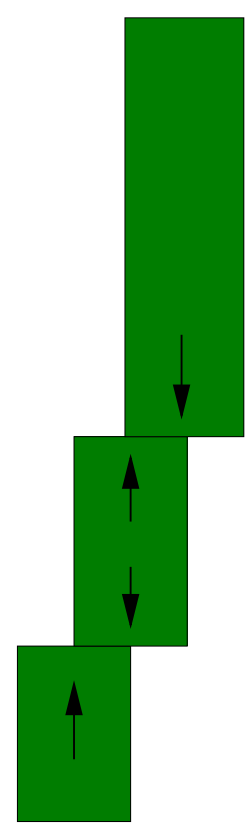

$\mathrm{C}$

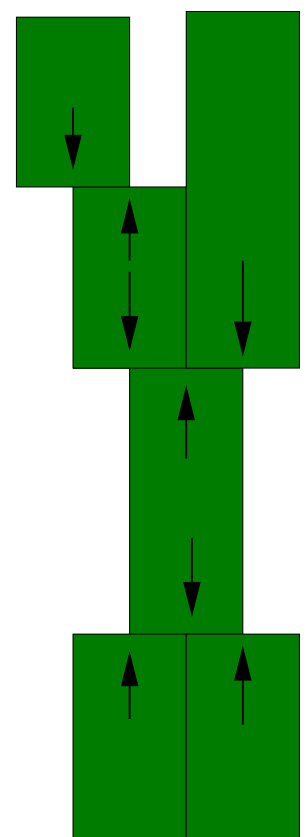

FIG. 2. Examples of higher order defects: A defect structure spanning $n+2$ columns is defined to be of order $n$. (A) A frustrated dead end : a defect of order one which spans three columns. (B) A defect of order two spanning four columns.(C) A defect of order three spanning five columns.

its downstairs neighbor is represented as shown in fig. 1 in the coarse grained picture. Taking the coarse grained view involves the loss of some specific information about the system. One loses information about the exact position of a nucleation point and also the exact shape of the doubling step. Positions in the vertical and horizontal direction can only be specified up to a single step width. Thus the coarse graining will also fail to capture the meandering of individual steps.

Once a nucleus has been formed the two steps will eventually form a double step unless it gets frustrated as described previously (and in fig. 1). If we examine the system after all evolution has ceased, there are thus at least two possible entities: a perfectly doubled step which occupies two columns of the coarse grained lattice and a frustrated pair which occupies three columns. There are in principle higher order defects that can occupy more columns (see fig. 2). For example one can have an extended structure where the doubling of steps 1 and 2 is frustrated by the doubling of steps 2 and 3 at another point which in turn is frustrated by steps 3 and 4 nucleating a doubling event and so on. To be able to proceed with an analysis we need to understand what kind of defects are important and under what conditions. We first consider the simplest case where the zippering rate is taken to be infinite.

\section{B. Infinite Zippering Rate}

In this case the nucleation rate, $I$, is assumed to be some finite number while the zippering rate, $Z$, is taken to be infinite. Now any nucleation will lead to a perfectly doubled step as the steps zipper together instantaneously. There will be no frustrated dead ends in this scenario. However there will be isolated steps, both of whose neighbors have doubled with other steps. The question then is how many of these isolated steps will be present in the final state when all evolution is complete.

To answer this question, we first note that a nucleation attempts the occupation of two columns in the coarse grained lattice picture. If the columns are unoccupied before the attempt, the nucleation is successful and both columns are occupied. Thus in any given time step when a succesful nucleation occurs two adjacent columns are fully occupied. Furthermore once these columns have been occupied subsequent successful nucleations cannot overlap either of these columns. We can now visualize the process as occuring on a 1D lattice each site of which corresponds to a column on our original lattice. A successful nucleation event leads to the occupation of two consecutive sites on this 1D lattice. 
Each nucleation attempt therefore corresponds to a random choice of a pair of consecutive sites. If both these sites are unoccupied, the attempt is successful and as a result both the sites are occupied. This process is exactly random sequential adsorption (RSA) of dimers (objects that occupy two lattice sites) onto a 1D lattice. In the RSA process a dimer attempts to adsorb onto the lattice at every time step, corresponding to the nucleation attempts in our system. Dimers cannot overlap just as in our system already doubled steps cannot double again with a different step. Thus in the limit of an infinite zippering rate our problem maps exactly to an RSA problem of dimers adsorbing onto a 1D lattice.

Though the solution to the RSA problem is standard [10,14], we present the solution for completeness and to set the stage for the next subsection. We first define $P_{n}$ to be the probability that a randomly chosen site is part of a sequence of at least $n$ consecutive empty sites. We can then write down the time evolution of this probability for $n \geq 1$

$$
\frac{d P_{n}}{d t}=-k(n-1) P_{n}-2 k P_{n+1}
$$

The first term accounts for the sequence being broken by the adsorption of a dimer within the $n$ sites, which can be done in $n-1$ ways. The second term refers to a dimer overlapping the sequence from either end if the sequence has at least $n+1$ sites. This can be done in two ways. In order to solve this set of equations, we first define the conditional probability $g_{n}=P_{n} / P_{n-1}$. Using equation 1, we can write down the set of equations satisfied by the $g_{n}$ 's.

$$
\frac{d g_{n}}{d t}=-k g_{n}-2 k\left(g_{n} g_{n+1}-g_{n}^{2}\right)
$$

Now at time $t=0$, the lattice is empty and all the $P_{n}$ 's are identically unity. This implies that all the $g_{n}$ 's are unity at $t=0$. Therefore the initial conditions tell us that all the $g_{n}$ 's satisfy the same equation at $t=0$ since the second term on the right hand side in equation 2 does not contribute. However this means that as time progresses the $g_{n}$ 's evolve in an identical fashion, all of them being equal, with the second term never contributing. Thus $g_{n}=f(t)$ is simply a function of time and does not depend on $n$. We can therefore look for solutions to equation 1 of the form

$$
P_{n}=f(t) P_{n-1}
$$

Using this with eq.(1) gives a differential equation for $f(t)$ (which can also obtained directly from eq.2) that may be solved to yield the ansatz

$$
P_{n}=e^{-k(n-1) t} P_{1}
$$

It may be readily verified that the ansatz consistently satisfies eq.(1). Now using (1) and (4) we get

$$
\frac{d P_{1}}{d t}=-2 k e^{-k t} P_{1}
$$

which yields

$$
\ln P_{1}=2 e^{-(k) t}+c
$$

where $c$ is an arbitrary constant. Using the initial condition that at $t=0$, the lattice is empty and hence $P_{1}=1$ we get

$$
c=-2
$$

We thus have an explicit solution for $P_{1}$

$$
P_{1}=\exp \left[2 e^{-(k) t}-2\right]
$$

This tells us that when $t \rightarrow \infty, P_{1} \rightarrow e^{-2}$. The fraction of sites that are unoccupied in the final state is therefore $e^{-2} \approx 13.5 \%$. Thus even in the case where we only have perfectly doubled steps the percentage of total area covered by the doubled steps is $86.5 \%$. There will always be $13.5 \%$ of the surface covered by isolated single step defects. In the case of a finite system we expect $13.5 \%$ defective area on average. We will later consider the case where the ratio $I / Z$ is small but finite. Here we expect not only isolated step edges but also frustrated dead ends and possibly defects of higher order. 


\section{Accounting for frustrated dead ends}

In real experimental situations $[2,3,11]$, one observes not only perfectly doubled steps but also frustrated dead ends (defects of order one). In this section we will lay down the mathematical framework, which we will use to treat the occurence of defects. Suppose we are in a regime (low $I / Z$ ) where we have only two significant entities: perfectly doubled steps and frustrated dead ends. A perfectly doubled step occupies two columns and a frustrated dead end spans three columns. We may now think of the process as occuring on a 1-D lattice with dimers (blocks of two lattice units) representing perfectly doubled steps and trimers (blocks of three lattice units) representing frustrated dead ends adsorbing onto the lattice at different rates. The dimer attempt rate is denoted by $k$ and the trimer attempt rate by $k^{\prime}$. These attempt rates are in general functions of time, the nucleation rate and zippering rate. The problem of determining these attempt rates will be dealt with in the next section. For now we take them to be arbitrary

functions of time. The kinetics of this process corresponds to the problem of random sequential adsorption (RSA) of a binary mixture $[12,13]$ though with attempt rates that are functions of time. As before we first define $P_{n}$ to be the probability that a randomly chosen site is part of a sequence of at least $n$ consecutive empty sites. We can then write down the time evolution of this probability for $n>2$

$$
\frac{d P_{n}}{d t}=-k(n-1) P_{n}-2 k P_{n+1}-k^{\prime}(n-2) P_{n}-2 k^{\prime} P_{n+1}-2 k^{\prime} P_{n+2}
$$

The first term accounts for the sequence being broken by the adsorption of a dimer within the $n$ sites, which can be done in $n-1$ ways. The second term refers to a dimer overlapping the sequence from either end if the sequence has at least $n+1$ sites. This can be done in two ways. The third, fourth and fifth terms are similar to the first and second except that they refer to the adsorption of trimers in an analogous fashion. Introducing the conditional probabilities $g_{n}=P_{n} / P_{n-1}$ and using the initial conditions, we find that $g_{n}=f(t)$ is only a function of time and does not depend on $n$. We therefore introduce the ansatz

$$
P_{n}=f(t) P_{n-1}
$$

Using this in conjunction with equation (9) and the initial condition $P_{n}=1$ yields

$$
f(t)=e^{-\int_{0}^{t} k\left(t^{\prime}\right)+k^{\prime}\left(t^{\prime}\right) d t^{\prime}}
$$

This along with equations (9) and (10) yield an explicit solution for $P_{2}$

$$
P_{2}=\exp \left[-\int_{0}^{t} k\left(t^{\prime}\right) d t^{\prime}-\int_{0}^{t}\left[2 f\left(t^{\prime}\right)\left(k\left(t^{\prime}\right)+k^{\prime}\left(t^{\prime}\right)\right)+2 k^{\prime}\left(t^{\prime}\right)(f(t))^{2}\right] d t^{\prime}\right]
$$

and hence from (10) for all $P_{n}$ for $n \geq 2$. For $n=1$ we have

$$
\frac{d P_{1}}{d t}=-2 k P_{2}-3 k^{\prime} P_{3}
$$

which upon integration gives

$$
1-P_{1}(t=\infty)=\int_{0}^{\infty}\left(2 k P_{2}+3 k^{\prime} P_{3}\right) d t
$$

$P_{1}(t=\infty)$ is simply the fraction of space occupied by isolated dead ends at asymptotic coverage. The two terms on the right are simply the fraction of space occupied by the dimers (double steps) and trimers (frustrated dead ends) respectively. It is to be noted that this constitutes an exact solution for the problem of random sequential adsorption of a binary mixture in 1D with arbitrary time dependence of the attempt rates. It is also to be noted that this analysis may be extended to include defects of higher order if one knows the attempt rates for all the species being considered. We now consider the case with $I / Z$ small but non-zero and compute the rates $k$ and $k^{\prime}$ explicitly in terms of the experimental parameters $I$ and $Z$.

\section{Nonzero but Small I/Z}

We now apply the formalism developed in the previous section to our problem where we are given the experimental parameters: the nucleation rate $I$ and the zippering rate $Z$. The case when $I / Z$ is small but non-zero is important as 


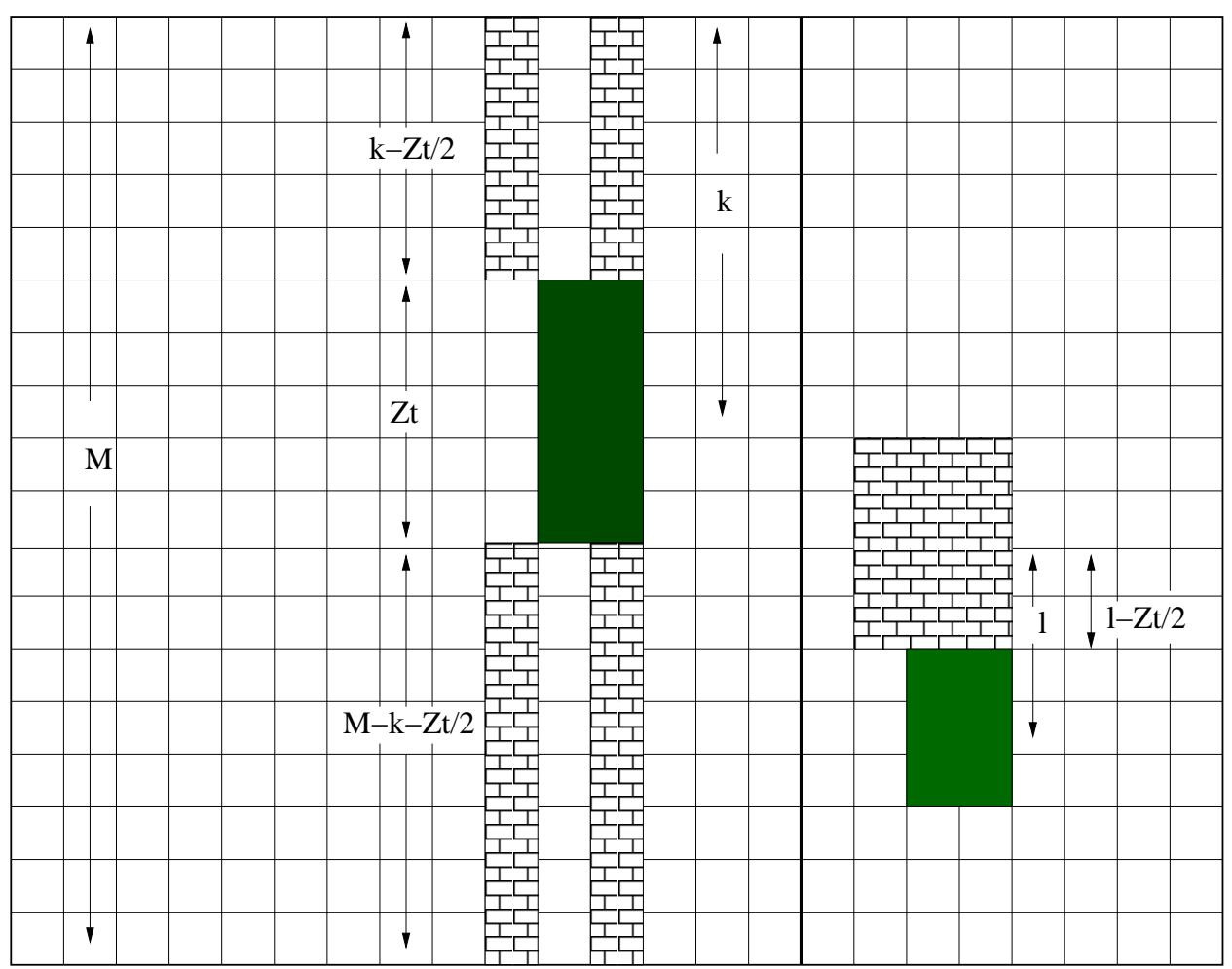

FIG. 3. Left : Scenario after a time $t_{i}=t\left(<t_{a}\right)$ after the first nucleation on an empty lattice. The nucleation occured $k$ lattice sites from the nearest horizontal edge. The doubled step (denoted by the shaded region) has grown to a length $Z t$. The sites where a nucleation would lead to frustration ( denoted by the "walled" sites ) number $2(M-Z t)$. Right : A situation after a time $t_{i}=t$ after a successful nucleation. "Walled" sites denote the places where a nucleation could lead to the growing doubled step being "cut-off" before it reaches a length $l_{0}=l$ on one side.

it is closer to reality. Most experimental situations have $I / Z$ values which are typically of order $10^{-3}$ to $10^{-1}[7,11]$. To begin our analysis we need to understand for what values of $I / Z$ different orders of defects become important. Intuitively one expects that for small enough $I / Z$, considering only defects of order one would be a good approximation. To estimate how small $I / Z$ needs to be we first consider the mean free path, $\langle l\rangle$, of a successful nucleation on an empty lattice. By mean free path we mean the average length to which the doubled step grows before it is cut off by other nucleations.

Heuristically one can say that higher order defects will not be important when the mean free path is much larger than the size of the lattice we are dealing with i.e. $\langle l\rangle \gg M$. We now focus on a regime of low $I / Z$ that satisfies the above condition with the assumption that we only have frustrated dead ends. The explicit condition will be worked out later in this section.

We can now view the whole process as occuring on a 1-D lattice of size $M$ corresponding to the problem of random sequential adsorption (RSA) of a binary mixture. In our system there are only dimers trying to adsorb onto the lattice except they may turn into a trimer if another dimer overlaps one of its units within the time it takes to zipper $M$ steps. We thus view this as a RSA process with the rate at which trimers try to adsorb determined by the probability, $q$, of the above mentioned overlap occuring. We now compute this probability explicitly in terms of $I$ and $Z$.

We first define $q$ to be the probability that a nucleation event leads to a perfectly doubled step on an empty lattice. If $I$ is the nucleation rate over the $M \times M$ sample then the probability of any one element initiating a nucleation in time $\Delta t$ is $I / M^{2} \Delta t$. Now consider a nucleation event $k$ lattice constants away from the nearest horizontal edge of the $M \times M$ lattice (see fig. 3).

Upto a time $t_{a}=2 k / Z$, both ends of the doubled step grow by zipping. For $t>t_{a}$ only one end grows since the other end has already reached the edge of the lattice. This part of the process takes a time $t_{b}=2(M-2 k) / Z$. For $t<t_{a}$ the number of sites where a nucleation can lead to frustration is $2(M-Z t)$. For $t_{a}+t_{b}>t>t_{a}$ the number of sites where a nucleation can lead to frustration is $2\left(M-2 k-Z\left(t-t_{a}\right) / 2\right)$. Now the probability at a time $t_{i}<t_{a}$ 
past a nucleation that the doubling steps do not get frustrated in the next $\Delta t$ of time is given by

$$
\begin{aligned}
P_{a}(i) & =\left(1-\frac{I}{M^{2}} \Delta t\right)^{2\left(M-Z t_{i}\right)} \\
& \approx \exp \left[-\frac{I}{M^{2}} 2\left(M-Z t_{i}\right) \Delta t\right]
\end{aligned}
$$

Similarly for $t_{a}+t_{b}>t_{j}>t_{a}$, we get

$$
\begin{aligned}
P_{b}(j) & =\left(1-\frac{I}{M^{2}} \Delta t\right)^{2\left(M-2 k-Z\left(t_{j}-t_{a}\right) / 2\right)} \\
& \approx \exp \left[-\frac{I}{M^{2}} 2\left(M-2 k-Z\left(t_{j}-t_{a}\right) / 2\right) \Delta t\right]
\end{aligned}
$$

Thus the total probability that the nucleation event, $k$ lattice constants from the edge, leads to a perfectly doubled step is

$$
\begin{aligned}
q_{k} & \approx \prod_{i} P_{a}(i) \prod_{j} P_{b}(j) \\
& \approx \prod_{i} \exp \left[-\frac{I}{M^{2}} 2\left(M-Z t_{i}\right) \Delta t_{i}\right] \prod_{j} \exp \left[-\frac{I}{M^{2}} 2\left(M-2 k-Z\left(t_{j}-t_{a}\right) / 2\right) \Delta t_{j}\right] \\
& =\exp \left[-\int_{t=0}^{t=t_{a}} \frac{I}{M^{2}} 2(M-Z t) d t-\int_{t=0}^{t=t_{b}} \frac{I}{M^{2}} 2(M-2 k-Z t / 2) d t\right] \\
& =\exp \left[-\frac{2 I}{Z}\right] \exp \left[-\frac{4 I}{Z}\left(\left(\frac{k}{M}\right)^{2}-\left(\frac{k}{M}\right)\right)\right]
\end{aligned}
$$

It is equally probable for the first nucleation to occur at any site along a column. We therefore have to average over all possible values of $k$ which are integers from zero to $M / 2$. This gives

$$
\begin{aligned}
q & =\left\langle q_{k}\right\rangle_{k} \\
& =\exp \left[-\frac{2 I}{Z}\right] \frac{2}{M} \sum_{k=0}^{k=M / 2} \exp \left[-\frac{4 I}{Z}\left(\left(\frac{k}{M}\right)^{2}-\left(\frac{k}{M}\right)\right)\right] \\
& \approx \exp \left[-\frac{2 I}{Z}\right]\left(2 \int_{0}^{1 / 2} \exp \left[-\frac{4 I}{Z}\left(x^{2}-x\right)\right] d x\right) \\
& =\exp \left[-\frac{I}{Z}\right]\left(\frac{Z}{I}\right)^{\frac{1}{2}} \frac{\pi^{\frac{1}{2}}}{2} \operatorname{erf}\left[\left(\frac{\mathrm{I}}{\mathrm{Z}}\right)^{\frac{1}{2}}\right]
\end{aligned}
$$

Thus we have a relation between the probability of a nucleation leading to a perfectly doubled step and the ratio of the nucleation and zippering rates. It is to be noted that to preserve invariance under rescaling time, $q$ can only depend on the ratio of the rates and not on their absolute magnitude. Happily, equation 26 respects this invariance.

We now return to the question of when the approximation of considering only order-one defects is valid. To do this, we compute the value of the mean free path of a successful nucleation, defined earlier, explicitly. We first compute the probability that the doubled step length on one side of the point of nucleation exceeds $l_{0}$. At any time $t_{i}$ after the nucleation event the probability that the doubling step does not get stopped by doubling events in its path in the next time step $(\Delta t)$ is given by

$$
\begin{aligned}
P(i) & =\left(1-\frac{I}{M^{2}} \Delta t\right)^{6\left(l_{0}-Z t_{i} / 2\right)} \\
& \approx \exp \left[-\frac{I}{M^{2}} 6\left(l_{0}-Z t_{i} / 2\right) \Delta t\right]
\end{aligned}
$$

Here we take the probability of not stopping the zippering end of the doubling step in the next $\Delta t$ of time to be the probability of having no nucleation in time $\Delta t$ at any site raised to the power of the number of sites where such a 
nucleation would lead to stoppage (see fig. 3). Now the total probability is simply the product of the $P_{i}$ 's which can be written as the exponential of an integral yielding

$$
P\left(l>l_{0}\right)=\exp \left[-\frac{6 I}{Z}\left(\frac{l_{0}}{M}\right)^{2}\right]
$$

Knowing this probability distribution we can calculate the mean length $\langle l\rangle$, which is what we defined as the mean free path. The mean free path is thus

$$
\langle l\rangle=2 \int_{0}^{\infty} \frac{d P}{d l} l d l=M\left(\frac{4 \pi}{6}\right)^{\frac{1}{2}}\left(\frac{Z}{I}\right)^{\frac{1}{2}}
$$

Using the explicit formula for $\langle l\rangle$ in terms of $I / Z$ above, the condition for neglecting higher order defects reduces to $I / Z \ll 4 \pi / 6 \sim 2.09$. As noted before for most realistic experimental situations this situation is easily satisfied. Simulation results presented later support our assumption.

Now, the value of $q$ derived above neglects the influence of nucleations other than the ones that can frustrate the initial zippering double step. For example a nucleation directly below and aligned with the original growing double step will block sites where nucleations could have frustrated the original growing double step. This is also true for nucleations on columns on either side of the original doubling step. In general a nucleation occuring many columns away may still influence the probability of the original nucleation forming a perfectly doubled step. Intuitively we would expect that the further such a column is from the original nucleation, the less its influence will be. Indeed for a nucleation occuring $j$ columns away the probablity that its effect will propagate to the column where the original nucleation took place will go as $(I / Z)^{j}$. This is because, for this to happen, we need $j$ nucleations (for each intervening column), each of which will occur roughly with a probability proportional to $I / Z$. Thus for small enough values of $I / Z$ we may neglect the effect of columns that are further away. However to be sure that this is not a big effect we need to ascertain the effect of nucleations on columns that are closest to the original doubling step. To do this, we need to take into account the probability that a site at which a nucleation could lead to frustration may not be available for occupation. Thus equation 16 will read

$$
P_{a}(i)=\left(1-\frac{I}{M^{2}} u \Delta t\right)^{2\left(M-Z t_{i}\right)}
$$

The extra factor $u$ reflects the probability that the site in question is available for occupation. $u$ may be roughly related in a "mean field" sense to the average fraction of unoccupied sites at that time. As an approximation we take this fraction to be $P_{1}$ for the case with an infinite zippering rate. Then going through the above analysis will yield a "corrected" value of $q$. We find that $q$ goes up by a factor which is about $6.2 \%$ when $q \sim 0.5$ and less than $0.1 \%$ when $q \sim 0.9$. Thus we find that neglecting these effects does not alter our results by much.

It is to be noted that when the only structures present were perfectly doubled steps $(Z=\infty)$, the mapping to the RSA problem was exact. Now we have two kinds of entities. The doubled steps still cannot be overlapped and are put down randomly. They are still amenable to RSA analysis. However the frustrated dead ends can become defects of order two (fig.2) when the sections of them that are still single steps merge with a neighboring step if available. If this occurs during the process when there are still pairs of single steps that can form perfectly doubled steps, it will affect the dynamics and hence the asymptotic fraction of different species. It is this process that we neglect as a first approximation. Later simulation results show that this is a reasonable assumption.

We now consider how the competition parameter $q$ is altered when the surrounding lattice is not empty as discussed above. The value of $q$ does depend on the environment in which the nucleation occurs. We can imagine three possible scenarios. (1) Columns on either side of the freshly nucleated double step are already occupied (doubled). In this case $q=1$, since this nucleation cannot be frustrated. (2) Columns on both sides are empty (single). In this case we may use the result for the empty lattice, since for low enough $I / Z$, as we argued before, the state of columns further away will not matter much. (3) One side of the freshly nucleated double step is occupied. The correct value to be used here will be $q^{1 / 2}$. The relative probabilities of these scenarios occuring will evolve as a function of time. In order to account for this we ought to use the more general treatment, where $q$ can be an arbitrary function of time, presented in the previous section. However since most of the defects form in the early stages, we anticipate that the asymptotic fraction of defects will not be altered significantly if we use a constant value of $q$, evaluated for the empty lattice. Our simulation results confirm this view. The dynamics will however will be sensitive to changes in $q$ and this will be addressed in the section on time dependence. For now we take $q$ to be a fixed constant throughout the process. 


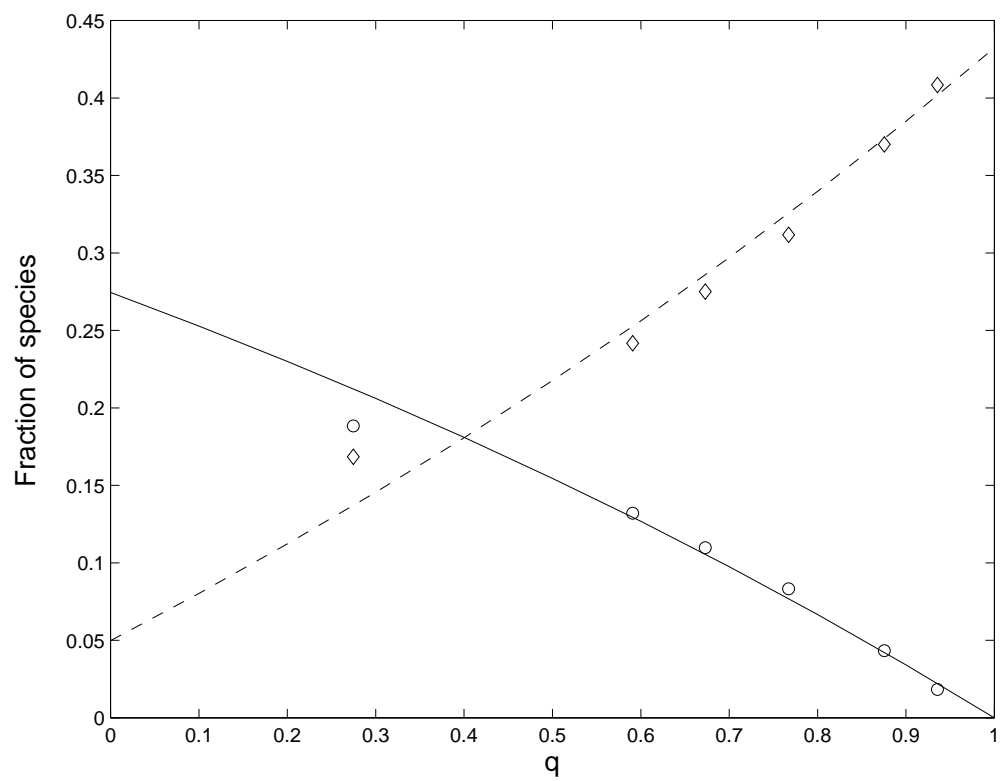

FIG. 4. Plots of fraction of doublets and triplets as a function of $q$. The fraction of a species is the number of that species divided by the size of the lattice ( 60 here). Solid and dashed curves are obtained by numerical integration of 34 and refer to the fraction of frustrated dead ends and perfectly doubled steps respectively. The simulation results for the fraction of frustrated dead ends (open circles) and perfectly doubled steps (diamonds) are also shown and are plotted with $q$ values computed using the equation (26)

We can now view the process as a RSA of a binary mixture of dimers and trimers on a 1-D lattice. A dimer adsorbs onto the lattice with a probability $q$ and a trimer with a probability $1-q$. If $k_{0}$ is the overall attempt rate at which nucleations are tried on the 1-D lattice then the dimers have an attempt rate $k=k_{0} q$ and the trimers have an attempt rate $k^{\prime}=k_{0}(1-q)$. We now use the results derived for the process on an infinite 1-D lattice with dimers attempting to adsorb at a rate $k$ and trimers at a rate $k^{\prime}$ (as in previous section). Using the fact that here $k$ and $k^{\prime}$ are constants eq.12 yields

$$
P_{2}=\exp \left[-k t+2 e^{-\left(k+k^{\prime}\right) t}+\frac{k^{\prime}}{k+k^{\prime}} e^{-2\left(k+k^{\prime}\right) t}-\left(2+\frac{k^{\prime}}{k+k^{\prime}}\right)\right]
$$

and hence from (10) an explicit solution for all $P_{n}$ for $n \geq 2$. For $n=1$ we have

$$
\frac{d P_{1}}{d t}=-2 k P_{2}-3 k^{\prime} P_{3}
$$

which upon integration gives

$$
1-P_{1}(t=\infty)=\int_{0}^{\infty}\left(2 k P_{2}+3 k^{\prime} P_{3}\right) d t
$$

$P_{1}(t=\infty)$ is simply the fraction of space occupied by isolated dead ends at asymptotic coverage. The two terms on the right are simply the fraction of space occupied by the dimers and trimers or double steps and frustrated dead ends respectively. Figure 4 shows a plot of the fraction of various species as a function of $q$. This is the only parameter in the problem as the asymptotic coverages are clearly independent of the overall rate $k_{0}$. Thus we have demonstrated that both the nature and number of defects in the final state depend only on the dimensionless parameter $q$. It is to be noted that the isolated single step population (not shown) does not change much $(8-13.5 \%)$ with $q$ in contrast to double steps and frustrated dead ends. This is in qualitative agreement with experimental results [11]. It is also to be noted that when $q \lesssim 0.58$, the fraction of width occupied by the defects exceeds that occupied by perfectly doubled steps. This characterization can be used to infer the ratio $I / Z$ by simply looking at the number of defects in the final structure. One thus gains information about the dynamics of the process from the final state. 


\section{E. Scaling of the fraction of defects}

A point of interest is the case where $Z \gg I$. Here the nucleations are the rate limiting step and the zippering occurs almost instantaneously. In this case $k^{\prime} \rightarrow 0$ and we retrieve the $Z=\infty$ case where we can explicitly solve for $P_{1}$ yielding $P_{1}(t=\infty)=e^{-2}$. Thus even in this situation $13.5 \%$ of the steps remain as isolated step defects.

In the limit of $q \rightarrow 1$ the mapping to the RSA problem becomes exact. We now consider the scaling of the number of frustrated dead ends with $\epsilon \equiv 1-q$ in this limit. The fraction of frustrated dead ends (defects of order one) is given by

$$
\begin{aligned}
p_{f r} & =\int_{0}^{\infty} k^{\prime} P_{3} d t \\
& =\int_{0}^{\infty} k_{0}(1-q) P_{3} d t \\
& =k_{0} \epsilon \int_{0}^{\infty} \exp \left[-k_{0} t\right] P_{2} d t \\
& =k_{0} \epsilon \int_{0}^{\infty} \exp \left[-k_{0} t\right] \exp \left[-k t+2 e^{-\left(k+k^{\prime}\right) t}+\frac{k^{\prime}}{k+k^{\prime}} e^{-2\left(k+k^{\prime}\right) t}-\left(2+\frac{k^{\prime}}{k+k^{\prime}}\right)\right] d t
\end{aligned}
$$

Here $k=k_{0}(1-\epsilon)$ and $k^{\prime}=k_{0} \epsilon$. Using these in the above expression and retaining terms to lowest order in $\epsilon$ we obtain

$$
\begin{aligned}
p_{f r} & =k_{0} \epsilon e^{-2} \int_{0}^{\infty} \exp \left[-2 k_{0} t+2 e^{-k_{0} t}\right] d t \\
& =\epsilon e^{-2} \int_{0}^{\infty} \exp \left[-2 x+2 e^{-x}\right] d x \\
& =0.284 \ldots \epsilon
\end{aligned}
$$

Thus for small $\epsilon$ the fraction of frustrated dead ends rises linearly with $\epsilon$ with a slope of 0.284 . Figure 5 shows a plot of values of $p_{f r}$ obtained from the simulations described in the section.III versus $\epsilon$. The error bars on the individual data points are about $5 \%$. The line plotted is a best fit line to the points and yields a slope of $0.28 \pm 0.01$ and an intercept of $0.0000 \pm 0.0002$. This agrees very well with our prediction and shows that $p_{f r}$ indeed rises linearly with $\epsilon$ with a slope of 0.28 . The observed number of frustrated dead ends thus gives us explicit information about the competition parameter $q$ and hence the experimental rates.

\section{F. Accounting for nucleations outside the field of view}

In the above analysis we assumed that all doubling occurred as a result of nucleations that occurred within the region of observation. Clearly this need not be the case. Nucleations that occur outside the region of observation can lead to doubled steps within this region. In a typical experimental situation for example one would be most likely to examine some subsection of the sample and take that subsection as being representative. We thus need to account for unobserved nucleations in order to make contact with experiment.

Most of the analysis done before still holds for this case. Nucleations occurring outside the field of view will increase the overall attempt rate (since there will be doubled steps formed by nucleations occurring outside and zippering in). They will also make it more probable for a given nucleation to be frustrated (since nucleations occurring outside can also frustrate doubling steps within the field of view). If we are interested only in the asymptotic fraction of defects, we do not need to consider changes in the overall attempt rate. The only difference will arise in computing the probability that a given attempt at a doubled step succeeds without being frustrated i.e. our competition parameter $q$. We take the region of observation to be an $M \times M$ lattice embedded in a larger lattice which is $3 M \times 3 M$. The choice is simply made based on convenience and for ease of comparison with simulations. First we recall the concept of a mean free path for a zippering double step. We defined this to be the average length to which a zippering double step can grow from the nucleation point without being hindered by a nucleation in its path. Taking into account the probability per unit time of a nucleation occuring in the path and the zippering speed it was shown that the 


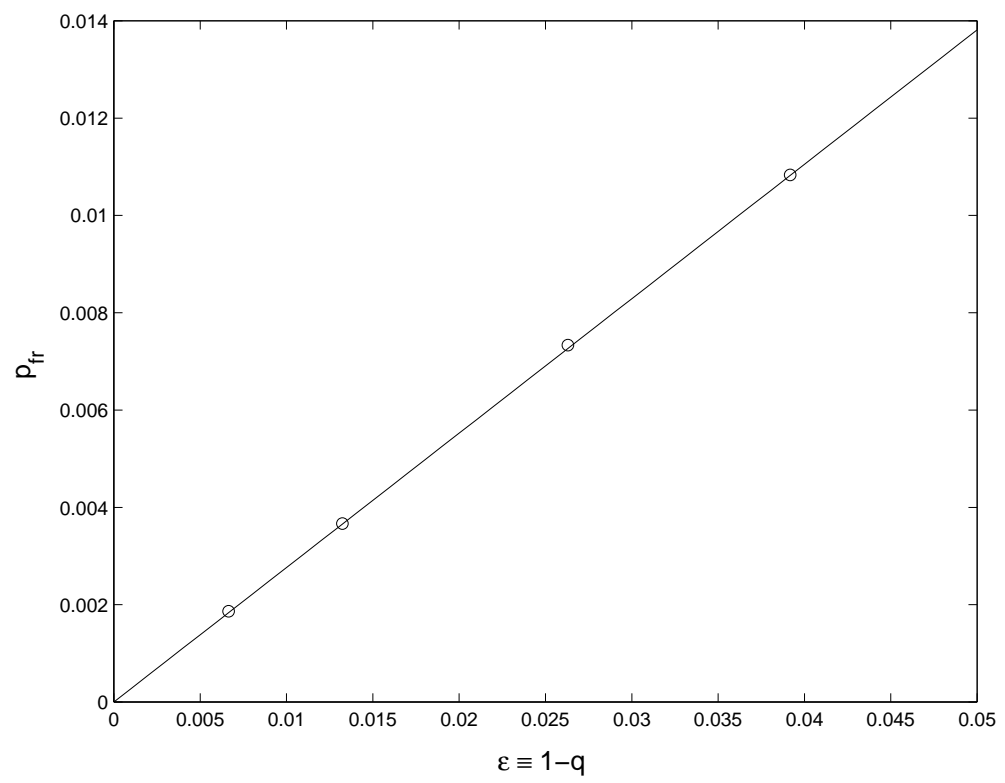

FIG. 5. Plot of fraction of frustrated dead ends versus $\epsilon \equiv 1-q$ showing the linear relation. The open circles are simulation results and the straight line is a best fit to these points. The best fit line has a slope of $0.28 \pm 0.01$ and an intercept of $0.0000 \pm 0.0002$.

probability of a zippering double step growing to a length greater than $l_{0}$ is

$$
p\left(l>l_{0}\right)=\exp \left[-\frac{6 I}{Z}\left(\frac{l_{0}}{M}\right)^{2}\right]
$$

where $I$ is the nucleation rate over an $M \times M$ area. Knowing this probability distribution we can calculate the mean length $\langle l\rangle$ which is what we defined as the mean free path. The mean free path is thus

$$
\langle l\rangle=M\left(\frac{4 \pi}{6}\right)^{\frac{1}{2}}\left(\frac{Z}{I}\right)^{\frac{1}{2}}
$$

Now we assume that the only nucleations that will have an effect on the region of observation occur either in this region or $\langle l\rangle$ above or below this region. This is because a zipper originating from a nucleation farther away is likely to be cut off by nucleations in its path. As before we consider the scenario at a time $t$ after a nucleation event inside the region of interest, $k$ lattice constants away from the nearest edge of the $M \times M$ lattice on an otherwise empty lattice. The probability that this nucleation leads to a perfectly doubled step may be derived in a manner analogous to the way we derived equation (22). There are two factors contributing to this probability. First there is the probability of being frustrated by nucleations inside the field of view. This is exactly the same as before. We denote this as $P_{i n}$, which is given by

$$
P_{\text {in }}=\exp \left[-\frac{2 I}{Z}\right] \exp \left[-\frac{4 I}{Z}\left(\left(\frac{k}{M}\right)^{2}-\left(\frac{k}{M}\right)\right)\right]
$$

This follows from eq.22. The second factor which we now consider, is to account for nucleations occurring outside the field of view. Now the probability at a time $t_{i}<t_{a}$ past a nucleation that the doubling steps do not get frustrated in the next $\Delta t$ of time is given by

$$
P_{\text {out }}^{1}(i)=\left(1-\frac{I}{M^{2}}\langle l\rangle P_{2}\langle p\rangle \Delta t\right)^{4}
$$

Here $t_{a}=2 k / Z$ as before. $\langle p\rangle$ refers to the average probability that a nucleation within a mean free path distance of the horizontal edge of the field of view, zippers to the edge without being cut off. This is simply the average of the probability given by eq.42 over this region.

$$
\langle p\rangle=\left(\frac{Z}{6 I}\right)^{1 / 2} \frac{M}{\langle l\rangle} \frac{\pi^{1 / 2}}{2} \operatorname{erf}\left[\left(\frac{Z}{6 I}\right)^{-1 / 2} \frac{\langle l\rangle}{M}\right]
$$


$P_{2}$ accounts for the probability that a nucleation is indeed possible at the site in question. We simply choose for $P_{2}$ the solution for the infinite zippering rate case (eqs. 8 and 4). The factor of 4 comes from the fact that there are four columns along which a nucleation from outside can zipper in to frustrate the original nucleation (see fig.3). We can write down a similar expression for times $t_{a}<t_{i}<t_{a}+t_{b}$ where $t_{b}=2(M-2 k) / Z$.

$$
P_{\text {out }}^{2}(i)=\left(1-\frac{I}{M^{2}}\langle l\rangle P_{2}\langle p\rangle \Delta t\right)^{2}
$$

The change here is in the exponent which changes from 4 to 2 , since once one end has reached the edge, there are only two columns left along which a nucleation from outside can zipper in to cause frustration. We now take the product $P_{\text {out }}^{1} P_{\text {out }}^{2}$ and integrate over time to get

$$
P_{\text {out }}=\exp \left[\frac{-4 I}{M^{2}}\langle l\rangle\langle p\rangle \int_{0}^{t_{a}} P_{2} d t\right] \exp \left[\frac{-2 I}{M^{2}}\langle l\rangle\langle p\rangle \int_{0}^{t_{b}} P_{2} d t\right]
$$

We similarly consider the probability that a nucleation event that occurs outside the region (within $\langle l\rangle$ of the edge of the region of interest) results in a perfectly doubled step.

$$
P^{0}=\exp \left[-\frac{2 I}{Z}\right] \exp \left[\frac{-2 I}{M^{2}}\langle l\rangle\langle p\rangle \int_{0}^{2 M / Z} P_{2} d t\right]
$$

This comes from putting $k=0$ in the product $P_{\text {in }} P_{\text {out }}$. This has to be weighted by the probability that the particular nucleation results in a zipper that makes it to the edge. This probability as calculated before (eq.42) is

$$
p(j)=\exp \left[-\frac{6 I}{Z}\left(\frac{j}{M}\right)^{2}\right]
$$

Now we simply average the probability of getting a perfectly doubled step over all positions (both inside and outside) with the appropriate weights following the steps to get eq.26. It is to be noted that if the distance from the edge of the field of view to the edge of the outer boundary is less than the mean free path, then $\langle l\rangle$ in the above expressions is to be replaced by this distance. The integrals cannot be performed to yield a closed form answer. These however can be numerically evaluated for specified values of $I / Z$. The dependence of the fraction of species on $q$ remains the same as before. Only the relation between $q$ and $I / Z$ has changed. Figure 6 shows a plot of the fraction of various species as a function of $q$. It is to be noted that, for the same value of $I / Z$, an open subsystem will have a smaller value of $q$ and hence a larger number of frustrated dead ends than a closed system of the same size (see fig.7). This is what one would intuitively expect.

One can use this analysis to infer the number and nature of defects in a larger sample simply by looking at a small patch and inferring the value of $I / Z$. However care must be taken if the calculated value of $I / Z$ for the larger sample exceeds about 0.5. One must then do an extended analysis incorporating a finite number of higher order defects dictated by the value of $I / Z$. It is to be noted that this treatment contains several ad hoc approximations and should only be taken as a rough estimate and a proposed methodology.

\section{G. Time Dependence}

Until now we have been looking at the asymptotic $(t \rightarrow \infty)$ limit of the surface morphology. In this non-equilibrium problem however the final morphology is dictated by the dynamic evolution and hence it is interesting to look at this evolution. Experimentally too there has been recently much progress in studying the time evolution of the step doubling process $[2-5]$.

We first introduce an order parameter $\psi$ which we define to be the fraction of sites that have undergone doubling. A completely empty matrix in which all steps are single corresponds to $\psi=0$ and a completely full state in which all sites are doubled corresponds to $\psi=1$. At the beginning $(t=0)$ we start with a situation where all the steps are single $(\psi=0)$. Now consider the situation after a time $t$. We define the average time it takes for a perfectly doubled step to form to be $t_{2}^{*}=3 M / 2 Z$. We assume it takes roughly half the time for a frustrated dead end to form and define $t_{3}^{*}=3 M / 4 Z$. We make these approximate estimates since we would just like to give a qualitative sense of the time dependence. Now all double steps initiated before time $t-t_{2}^{*}$ and all frustrated dead ends initiated before time $t-t_{3}^{*}$ have formed completely. Entities initiated within this interval have formed partly. So we have 


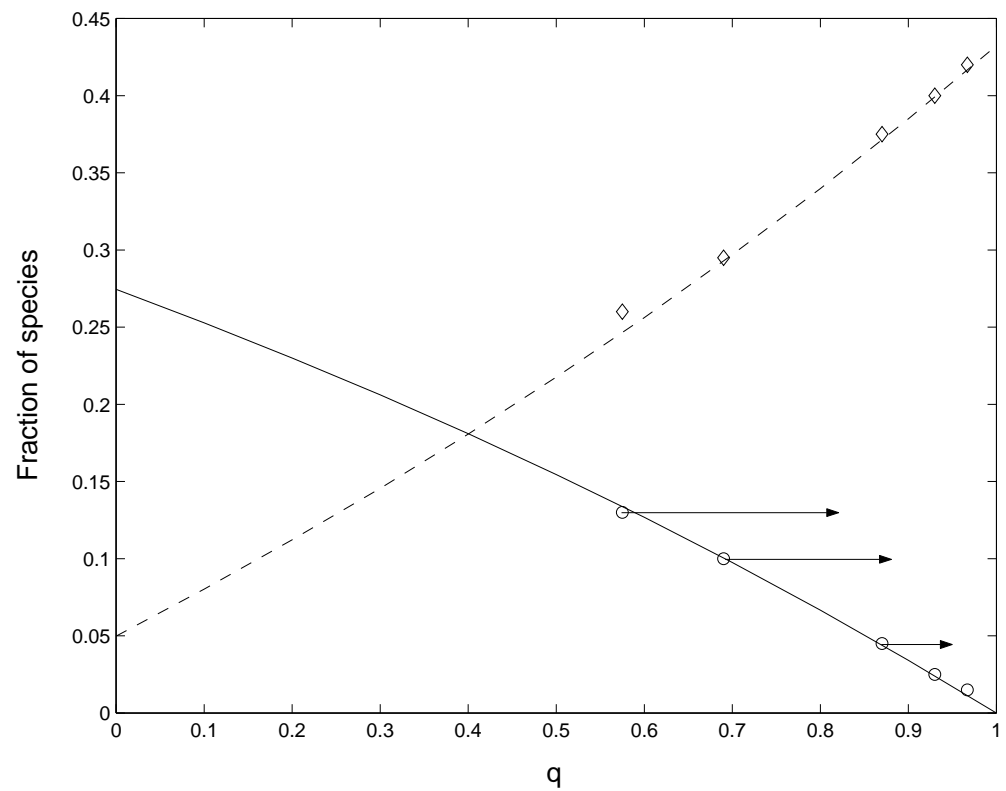

FIG. 6. Plots of fraction of doublets and triplets as a function of $q$. Solid and dashed curves are theoretical curves and refer to the fraction of frustrated dead ends and perfectly doubled steps respectively. These are identical to the curves in fig. 4 The simulation results for the fraction of frustrated dead ends (open circles) and perfectly doubled steps (diamonds) are also shown but with $q$ being computed using the relation for the case of an open subsystem. The arrows on selected data points indicate how much they would shift if $q$ were calculated using the relation for the case of a closed system. The fraction of a species is the number of that species divided by the size of the lattice (20 here).

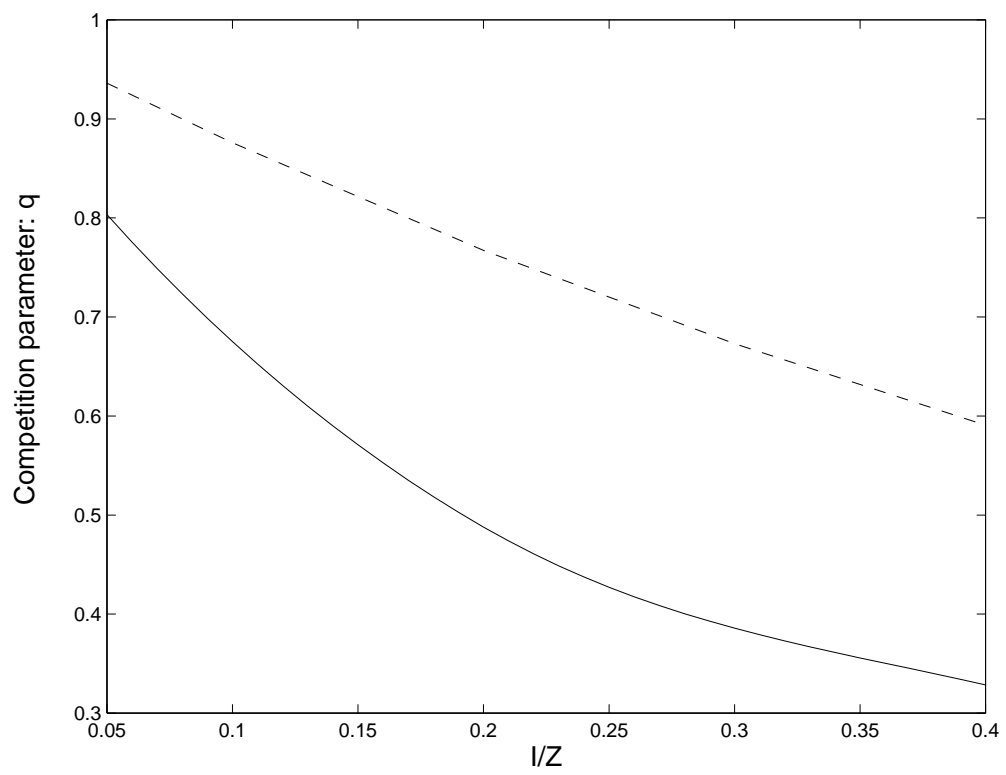

FIG. 7. Competition parameter versus $I / Z$ for the closed system (dashed line) and the open subsystem (solid line). Values of $q$ are lower for the open subsystem case for the same values of $I / Z$. 


$$
\psi(t)=\int_{0}^{t-t_{2}^{*}} 2 k P_{2} d t^{\prime}+\int_{0}^{t-t_{3}^{*}} 2 k^{\prime} P_{3} d t^{\prime}+\int_{t-t_{2}^{*}}^{t} 2 k^{\prime} P_{2} \frac{t-t^{\prime}}{t_{2}^{*}} d t^{\prime}+\int_{t-t_{3}^{*}}^{t} 2 k^{\prime} P_{3} \frac{t-t^{\prime}}{t_{3}^{*}} d t^{\prime}
$$

The first two terms account for the completely formed (completed double steps and frustrated dead ends) structures. The third and fourth terms account for the structures that are partly formed. The factor of two in all the terms comes from the fact that each doubled step occupies two columns. The frustrated dead ends also effectively occupy two columns (in terms of area) though they span three columns. Since we have explicit solutions for $P_{2}$ and $P_{3}$ as functions of time we can compute $\psi(t)$. The only parameter we have not yet evaluated is the overall attempt rate $k_{0}$. We may think of each doubled step as being caused by one nucleation and each frustrated dead end being caused by two. Then the attempt rate of dimers plus twice the attempt rate of triplets ought to be equal to the nucleation rate per column.

$$
\begin{aligned}
k+2 k^{\prime} & =k_{0} q+2 k_{0}(1-q) \\
& =k_{0}(2-q)=\frac{I}{M}
\end{aligned}
$$

which yields

$$
k_{0}=\frac{I}{M(2-q)}
$$

Having evaluated $k_{0}$ we can now compute $\psi(t)$ explicitly in terms of $I, Z$ and $M$. Figure 8 shows a plot of the computed $\psi(t)$ (dashed line) as a function of time for two different values of $I / Z(0.05$ and 0.1$)$. $Z=2$ in both cases. The difference between the two curves shows that the dynamics is quite sensitive to the parameters. Thus combining the information about the defects in the asymptotic structure and a measurement of the time dependence of $\psi(t)$ will allow us to uniquely determine both the nucleation and zippering rates. The mismatch between the analytical and simulation curves at late times was anticipated before and comes from the assumption of a constant $q$. To fix this, we use the solution for the case with the time dependent competition parameter, $q(t)$. To do this, we need to compute $q(t)$. Now, the value of $q$ depends on where a particular nucleation event takes place. As mentioned before, if the nucleation takes place at a site which belongs to a set of two empty sites with doubled steps on either side, the value of $q$ is unity since this doubling step cannot be frustrated. This situation would occur with a probability $1-P_{3}$. This includes the probability of picking isolated single steps where nucleations are not possible, resulting in the attempt being rejected. As a first approximation we assume that nucleation attempts at all other places (with probability $P_{3}$ ) have the value of $q$ computed for an empty lattice, $q_{0}$. In principle we can systematically refine this approximation by considering sites adjacent to a double step but belonging to a large set of consecutive empty sites having a different value of $q$ and so on. However we find that the first level of approximation is sufficient for our purposes. Thus we take the competition parameter to have a time dependent value

$$
q(t)=q_{0} \times\left(P_{3}\right)+1 \times\left(1-P_{3}\right)
$$

We now solve the set of equations (12), (55) and (54) self-consistently by an iteration procedure starting with the solution for the case with a constant competition parameter, $q_{0}$. The results after a couple of iterations are plotted in 8 as solid lines. One immediately sees that these agree with the simulation points much better at later times. Thus our treatment allows us to capture the time evolution of the surface morphology fairly accurately.

\section{SIMULATION}

We now describe the simulation that produced the data in figures $2-5$. This simulation uses the coarse-grained representation of figure 1 but makes none of the assumptions leading to the curves in figures $2-5$. It thus serves as a test of our RSA approximations. We use an $M \times M$ matrix to mimic a square section of the sample. Every column of the matrix is regarded as a step. We have two experimental parameters that we may use: the nucleation rate $I$ and the zippering rate $Z$. A typical simulation cycle is as follows:

(1)A randomly chosen site of the matrix is addressed.

(2) If a chosen site is unoccupied and its neighbor to the right is also unoccupied these two sites are allowed to combine 


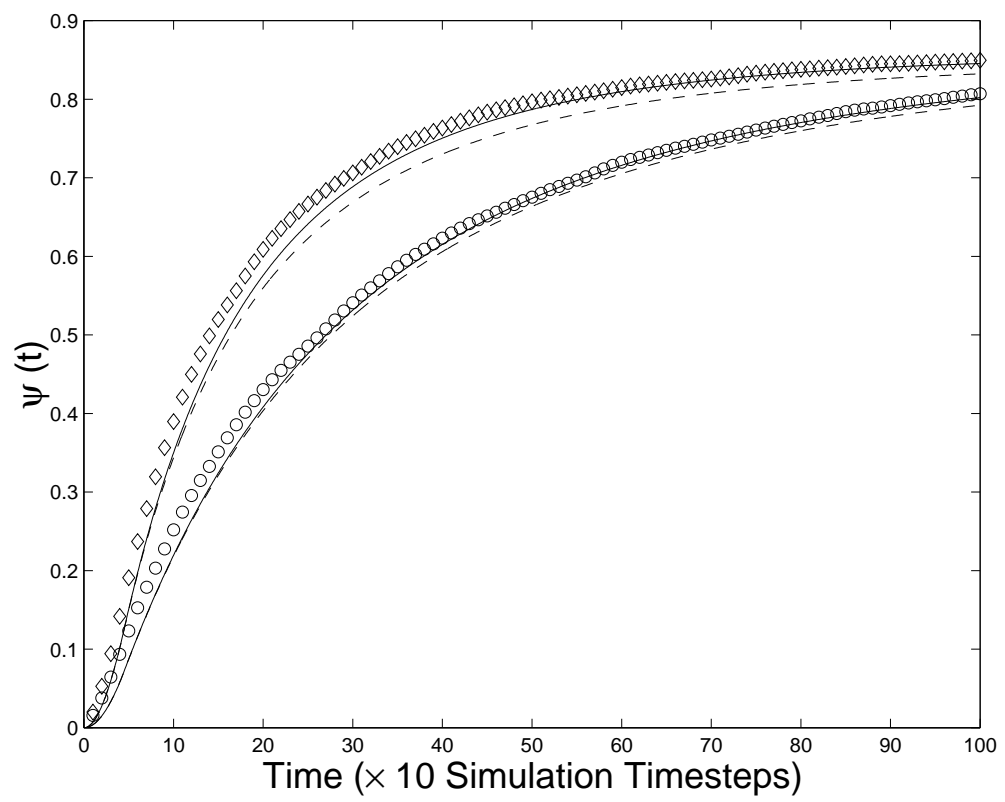

FIG. 8. Plots of $\psi(t)$ versus time for two different values of $I / Z$ (0.05 (lower curve) and 0.1 (upper curve)). The solid curves are obtained by numerical evaluation of 51 for the case with a time dependent competition parameter $q(t)$. Dashed curves are obtained by numerical evaluation of 51 for the case with a time independent competition parameter $q_{0}$. Simulation results obtained for $I / Z=0.05$ (open circles) and $I / Z=0.1$ (diamonds) are also shown.

to form a nucleus with a probability $p=I / M^{2} Z$. This takes care of the relative rates of nucleation and zippering. Now these two sites are considered occupied.

(3) Steps (1) and (2) are repeated making sure sites are not addressed twice until all sites of the matrix have been addressed.

(4) All steps that have previously initated doubling are allowed to grow by one lattice unit at each free end, if possible.

(5) Steps (1)-(4) are repeated until no new nucleations are possible and the surface is comprised only of perfectly doubled steps, frustrated dead ends and isolated single steps.

(5) The results are averaged over hundreds of runs for different values of the ratio $I / Z$.

\section{A. Final State}

The size of the matrix used was $M=60$ since the STM experiment described in [11] has a field of view roughly 60 terraces wide and 60 terraces tall. In the range of parameter values we looked at the number of defects that were not simple frustrated dead ends were few ( $\sim 15 \%$ of the defects for $q=0.5$ and decreasing with increasing $q$ ), thus justifying the rationale for our theoretical assumption. When these were encountered they were decomposed into constituent dead ends and counted as that many effective frustrated dead ends. For example an order two defect was counted as one frustrated dead end and one isolated step. Figure 4 plots the results of the simulation for various values of $I / Z$. We see very good agreement between the simulation and values predicted by theory for small $I / Z$. We also see that the theory begins to break down for large values of $I / Z(>0.46)$. It is to be noted that the largest number of perfectly doubled steps occurs in the limit of $Z \gg I$ and is about $43.75 \%$. This is consistent with our prediction for the $Z=\infty$ case.

We also consider the case where the region of interest is part of a larger region. We utilize the same $M=60$ matrix and we now count the defects and doubled steps in the central $20 \times 20$ submatrix. Figure 6 plots the results of the simulation for various values of $I / Z$. The agreement between the theoretical curve and the simulation values appear to be quite good in this case too. 


\section{B. Time dependence}

We also look at the evolution of the order parameter $\psi$ during a simulation run. The simulation is done on a $60 \times 60$ matrix as before. The fraction of sites that are occupied (doubled ) is registered after every 10 simulation timesteps. This data is recorded for hundreds of whole simulation runs. For each block of 10 timesteps we then record the average $\psi$ over all the runs. Figure 8 shows a plot of the averaged evolution of the order parameter as a function of simulation time for two different values of $I / Z$. We see that the simulation data and the theoretical curves agree quite well. It is to be noted that there are no adjustable parameters.

Thus overall the simulation results are in agreement with the theory and the theory effectively captures both the dynamics and the details of the asymptotic surface morphology.

\section{DISCUSSION OF RESULTS}

We now compare our results to experimental data so as to be able draw some physical conclusions. In the experiments by Wang et al [11] it was noted that under certain optimal conditions, a $100 \mathrm{~nm}$ by $100 \mathrm{~nm}$ section of the sample exhibited 5-6 frustrated dead ends after structural evolution had reached an asymptotic stage. The step zippering rate was measured to be $3.7 \AA s^{-1}$. The case to which this data ought to be compared is the one where the region of interest is embedded in a larger region. From figure 6 we immediately see that to get 5-6 frustrated dead ends in a $60 \times 60$ matrix we require $q \approx 0.7$. This gives us a value for the ratio $I / Z \approx 0.12$. Knowing the experimentally measured zippering rate we can also deduce the true nucleation rate. In our model we have $Z=2$ measured in units of step width per simulation timestep. The step width is about $1.65 \mathrm{~nm}$ which tells us that each simulation timestep corresponds to $2 \times 1.65 / 0.37 \approx 9$ seconds. Thus the true nucleation rate in this case would be $0.12 / 9=0.0133$ nucleations per second over the $100 \mathrm{~nm}$ by $100 \mathrm{~nm}$ section of the sample. Our analysis hence helps pin down the true experimental parameters simply by looking at the defects in the asymptotic stage.

Our analysis of the dynamics also gives us more useful insights. In particular we notice that $P_{3} \sim \exp (-\mathrm{kt}) \mathrm{P}_{2}$ (from eq.(10)). This tells us that the rate of defect formation drops exponentially faster than the rate at which perfectly doubled steps form. Often one wishes to minimize the number of defects. The way to do that would be to have a very low nucleation rate (for a given zippering rate). However then reaching the final state would take a very long time. Since we anticipate that most of the defects will be formed in the intial stages we could start with a low nucleation rate and after some time jump to a much higher rate. This would mean reaching the final state much faster with only a small increase in the number of defects. As an example we ran a simulation with $I / Z=0.05$ on a $M=60$ lattice. The number of defects was roughly one and it took 3600 timesteps to go to completion. A run where we started with the same value of $I / Z$ and then switched to a value 20 times higher $(I / Z=1)$ after 500 timesteps yielded two defects and took only 700 timesteps to complete. In contrast if we run the simulation for $I / Z=1$ from the beginning we get approximately 12 defects. Thus we gained a factor of 5 in time for a minor increase in the number of defects. One can also imagine trying different time dependent protocols to optimize the number of defects and the time.

Our prediction for the dynamic evolution of the order parameter (figure 8) is qualitatively similar to experimental data by Niu et al [4]. The authors use a phenomenological approach to fit their data using second order rate kinetics. Though the fit is good the analysis neglects that the fact that only neighboring steps can double. Another approach by Khare et al [6] analyzes the dynamics in terms of first passage times of random walkers using a fit with three adjustable parameters. However as the authors note, they do not take into account the formation of defects like the isolated steps and frustrated dead ends. Our analysis allows us to inspect the dynamics with no adjustable parameters if we first extract the relevant parameters from an inspection of the asymptotic structure. It also takes into account the formation of defects and their effect on the subsequent dynamics. This cannot be ignored for a manifestly non-equilibrium problem such as this.

There are however several details which we have ignored in this analysis. Firstly it was noticed [3] that zippering occurs much more slowly when the steps are surrounded by already doubled steps. Secondly we have not taken into account correlations between neighboring zippers. Another avenue of interest would be to integrate the time distribution of nucleation events postulated by Khare et al [6] with our RSA analysis. One could also incorporate higher order defects by a straightforward elaboration of our treatment. Thus it appears that our RSA approach will be applicable to a a range of such propagating surface reconstruction processes. Though we know of only one such process at the moment, many others are sure to emerge as atomic scale knowledge of adsorption on solid surfaces improves. 


\section{CONCLUSION}

We have shown here how emergent features of the novel step-doubling process can be quantitively understood. Our approximation of the process as a form of random sequential absorption leads to successful predictions in regions of experimental interest. The approximation permits simple analysis, yet it shows that naive analysis based on equilibrium statistics is misleading. The inadequacy of an equilibrium treatment is further apparent in the timedependence of our results. Changing the growth conditions over time can have a striking effect on the final state.

We have focussed on predicting the incidence of a particular type of defect: the frustrated dead end. But the method gives a way of understanding higher-order composite defects as well. Experimental mastery of propagating surface self-organization such as step doubling will improve over time. Along with this improvement, we expect stochastic models like the present one to be valuable guides in achieving desired structures.

\section{ACKNOWLEDGEMENTS}

The authors would like to thank V. Belyi, Y. Wang and S.J. Sibener for useful discussions. AG would also like to thank R. Bao, L. Tseng and E. Yuzbashyan for engaging conversations on the topic. This work was supported by the National Science Foundation via its MRSEC program under award number DMR 0213745.

[1] B. Lang, R.W. Joyner and G.A. Somorjai, Surf. Sci., 30, 454 (1972).

[2] T.P. Pearl and S.J. Sibener ,J. Chem. Phys., 115, 1916 (2001).

[3] T.P. Pearl and S.J. Sibener ,J. Phys. Chem. B, 105, 6300 (2001).

[4] L. Niu, D.D. Koleske, D.J. Gaspar, S.F. King and S.J. Sibener, Surf. Sci. , 356, 144 (1996).

[5] G. Hoogers and D.A. King, Surf. Sci. ,286, 306 (1993).

[6] S.V. Khare, T.L. Einstein and N.C. Bartelt, Surf. Sci. ,339, 353 (1995)

[7] K. Sudoh, H. Iwasaki and E.D. Williams , Surf. Sci. ,452, L287 (2000)

[8] M. Kardar, G. Parisi and Y.C. Zhang, Phys. Rev. Lett. ,56, 889 (1986)

[9] N.T.J. Bailey, Springer Lecture Notes in Biomathematics, 38, 233 (Springer, Berlin 1980).

[10] J.W. Evans, Rev. Mod. Phys. ,65, 1281 (1993)

[11] Yi Wang, T.P. Pearl, S.B. Darling, J.L. Gimmell and S.J. Sibener, J. App. Phys, 91, 2212 (2002).

[12] B. Bonnier, Europhys. Lett. , 18, 297 (1992).

[13] M.C. Bartelt and V. Privman, Phys. Rev. A , 44, R2227 (1991).

[14] P.J. Flory, J. Am. Chem. Soc. , 61, 1518 (1939). 